\title{
Correction to: Evaluating the effects of the novel GLP-1 analogue liraglutide in Alzheimer's disease: study protocol for a randomised controlled trial (ELAD study)
}

Grazia Daniela Femminella ${ }^{1 \dagger}$, Eleni Frangou ${ }^{2 \dagger}$, Sharon B. Love ${ }^{2}$, Gail Busza $^{1}$, Clive Holmes ${ }^{3}$, Craig Ritchie ${ }^{1}$ Robert Lawrence ${ }^{4}$, Brady McFarlane ${ }^{3}$, George Tadros ${ }^{5}$, Basil H. Ridha ${ }^{6}$, Carol Bannister ${ }^{7}$, Zuzana Walker ${ }^{8}$, Hilary Archer ${ }^{9}$, Elizabeth Coulthard ${ }^{9}$, Ben R. Underwood ${ }^{10}$, Aparna Prasanna ${ }^{11}$, Paul Koranteng ${ }^{12}$, Salman Karim ${ }^{13}$, Kehinde Junaid ${ }^{14}$, Bernadette McGuinness ${ }^{15}$, Ramin Nilforooshan ${ }^{16}$, Ajay Macharouthu ${ }^{17}$, Andrew Donaldson ${ }^{18}$, Simon Thacker ${ }^{19}$, Gregor Russell ${ }^{20}$, Naghma Malik ${ }^{21}$, Vandana Mate ${ }^{22}$, Lucy Knight ${ }^{23}$, Sajeev Kshemendran ${ }^{24}$, John Harrison ${ }^{25,26}$, Christian Hölscher ${ }^{27}$, David J. Brooks ${ }^{1,28}$, Anthony Peter Passmore ${ }^{15}$, Clive Ballard ${ }^{7}$ and Paul Edison ${ }^{1,29^{*}}$

Correction to: Trials (2019) 20: 191 https://doi.org/10.1186/s13063-019-3259-x

After publication of our article [1] the authors have notified us that the name of Prof. Christian Hölscher had been inadvertently forgotten when compiling the list of authors. Dr. Hölscher developed the concept of testing liraglutide in Alzheimer's mouse model, has done the preclinical experiments, and is a co-applicant of the Alzheimer Society UK grant that partially funds the study.

\section{Author details \\ ${ }^{1}$ Department of Medicine, Imperial College London, London, UK. ${ }^{2}$ Centre for Statistics in Medicine, Nuffield Department of Orthopaedics, Rheumatology and Musculoskeletal Sciences, University of Oxford, Oxford, UK. ${ }^{3}$ Southern Health NHS Foundation Trust, Havant, UK. ${ }^{4}$ SW London and St George's Mental Health Trust, London, UK. ${ }^{5}$ Aston Medical school, Aston University, Birmingham, UK. ${ }^{6}$ Brighton and Sussex University Hospitals NHS Trust, Brighton, UK. ' ${ }^{7}$ South London and Maudsley NHS Foundation Trust, London, UK. ${ }^{8}$ University College London and Essex Partnership University NHS}

The original article can be found online at https://doi.org/10.1186/s13063019-3259-x

* Correspondence: paul.edison@imperial.ac.uk

${ }^{\dagger}$ Grazia Daniela Femminella and Eleni Frangou contributed equally to this work.

'Department of Medicine, Imperial College London, London, UK

${ }^{29}$ School of Medicine, College of Biomedical and Life sciences, Cardiff University, Cardiff CF14 4YS, UK

Full list of author information is available at the end of the article
Foundation Trust, Runwell, UK. ${ }^{9}$ North Bristol NHS Trust, Bristol, UK. ${ }^{10} \mathrm{Cambridgeshire} \mathrm{and} \mathrm{Peterborough} \mathrm{NHS} \mathrm{Foundation} \mathrm{Trust,} \mathrm{Peterborough,}$ UK. "'Black Country Partnership NHS Foundation Trust, West Bromwich, UK. ${ }^{12}$ Northamptonshire Healthcare NHS Foundation Trust, Kettering, UK. ${ }^{13}$ Lancashire Care NHS Foundation Trust, Preston, UK. ${ }^{14}$ Nottinghamshire Healthcare NHS Foundation Trust, Nottingham, UK. ${ }^{15}$ Centre for Public Health, Queen's University Belfast, Belfast, UK. ${ }^{16}$ Surrey and Borders Partnership NHS Foundation Trust, Leatherhead, UK. ${ }^{17} \mathrm{NHS}$ Ayrshire and Arran, Crosshouse, UK. ${ }^{18}$ NHS Lanarkshire, Glasgow, UK. ${ }^{19}$ Derbyshire Healthcare NHS Foundation Trust, Derby, UK. ${ }^{20}$ Bradford District Care NHS Foundation Trust, Bradford, UK. ${ }^{21} 5$ Boroughs Partnership NHS Foundation Trust, Warrington, UK. ${ }^{22}$ Cornwall Partnership NHS Foundation Trust, Redruth, UK. ${ }^{23}$ Somerset Partnership NHS Foundation Trust, Bridgwater, UK. ${ }^{24}$ South Staffordshire and Shropshire Healthcare NHS Foundation Trust, Stafford, UK.

${ }^{25}$ Alzheimer Center VUmc Amsterdam, Amsterdam, the Netherlands.

${ }^{26}$ Institute of Psychiatry, Psychology \& Neuroscience King's College London, London, UK. ${ }^{27}$ Research Department, Henan University of Chinese Medicine, Zhengzhou, China. ${ }^{28}$ Newcastle University, Newcastle upon Tyne, UK.

${ }^{29}$ School of Medicine, College of Biomedical and Life sciences, Cardiff University, Cardiff CF14 4YS, UK.

Published online: 19 July 2020

\section{Reference}

1. Femminella, et al. Evaluating the effects of the novel GLP-1 analogue liraglutide in Alzheimer's disease: study protocol for a randomised controlled trial (ELAD study). Trials. 2019;20:191. https://doi.org/10.1186/ s13063-019-3259-x.

C The Author(s). 2020 Open Access This article is licensed under a Creative Commons Attribution 4.0 International License, which permits use, sharing, adaptation, distribution and reproduction in any medium or format, as long as you give appropriate credit to the original author(s) and the source, provide a link to the Creative Commons licence, and indicate if changes were made. The images or other third party material in this article are included in the article's Creative Commons licence, unless indicated otherwise in a credit line to the material. If material is not included in the article's Creative Commons licence and your intended use is not permitted by statutory regulation or exceeds the permitted use, you will need to obtain permission directly from the copyright holder. To view a copy of this licence, visit http://creativecommons.org/licenses/by/4.0/ The Creative Commons Public Domain Dedication waiver (http://creativecommons.org/publicdomain/zero/1.0/) applies to the data made available in this article, unless otherwise stated in a credit line to the data. 